Research Article

\title{
The Mechanical Behavior of a Multispring System Revealing Absurdity in the Relativistic Force Transformation
}

\author{
Mohammad Javanshiry \\ Independent Researcher, Tehran, Iran \\ Correspondence should be addressed to Mohammad Javanshiry; m.javanshiry1986@gmail.com
}

Received 8 September 2021; Revised 3 November 2021; Accepted 20 November 2021; Published 11 December 2021

Academic Editor: Theodore E. Simos

Copyright ( 2021 Mohammad Javanshiry. This is an open access article distributed under the Creative Commons Attribution License, which permits unrestricted use, distribution, and reproduction in any medium, provided the original work is properly cited.

The mechanical motion of a system consisting of simple springs is investigated from the viewpoint of two inertial observers with a relativistic relative velocity. It is shown that the final displacement of the springs is not measured the same by the observers. Indeed, it is demonstrated that there is an incompatibility between kinematics and dynamics in Einstein's relativity regarding the force transformation.

\section{Introduction}

This article represents an advanced version of the author's spring paradox [1] in which it was shown that the final displacement of two relatively moving springs is measured differently from the standpoint of different observers as soon as the springs meet each other. Here, we try to make the possible effect of the signal delay due to the constancy of light speed of little or no consequence as a cornerstone in resolving the paradox.

Similar to our previous works on the subject $[1,2]$, we insist here, too, that the relativistic dynamics are not easily reconcilable to the relativistic kinematics since there are fundamental deficiencies with the Lorentz transformation for force. Moreover, it is worthwhile to note that some other works show paradoxes of special relativity regarding rotating reference systems for only kinematic effects [3], which is related to the subject of this article.

Although the analysis demonstrated in the article is based on the well-known dynamics of special relativity, other dynamics have been introduced in some references of the literature. For instance, it has been shown that different dynamics can be derived for the kinematics of special relativity [4], and thus, our multispring system paradox analysis can be performed under other dynamics too. It is interesting whether the shown paradox holds for all possible dynamics.
In addition, the studies in $[5,6]$ develop new mathematical formalisms on special relativity, and hence, some theoretical research may investigate the application of these formalisms to the analysis of paradoxes such as the paradox discussed in this article.

On the other hand, there are alternative theories for special relativity $[7,8]$, and the continuation of the research presented in this article may concern checking whether the multispring system paradox also applies to these theories.

\section{The Multispring System Paradox}

Too many very thin identical springs, each with a similar constant of $k_{p}^{\prime \prime}$, are attached at one end to the circumference of a thin solid cylindrical plate, all being perpendicular to the plane that passes through the plate, and in the other end, the springs touch the floor. Since these springs are fused to the thin plate, we denote them by $P$. Another spring $S$ with a greater spring constant of $K_{S}^{\prime}$ attaches the center of the plate to the ceiling of the compartment in which the experiment is carried out (see Figure 1).

The distance between the floor and ceiling, as well as the free lengths of the springs, is $d_{0}^{\prime}$. On the other hand, it is assumed that the constants of the springs have the following relation:

$$
k_{P}^{\prime \prime}=\frac{K_{S}^{\prime}}{n^{\prime}}
$$




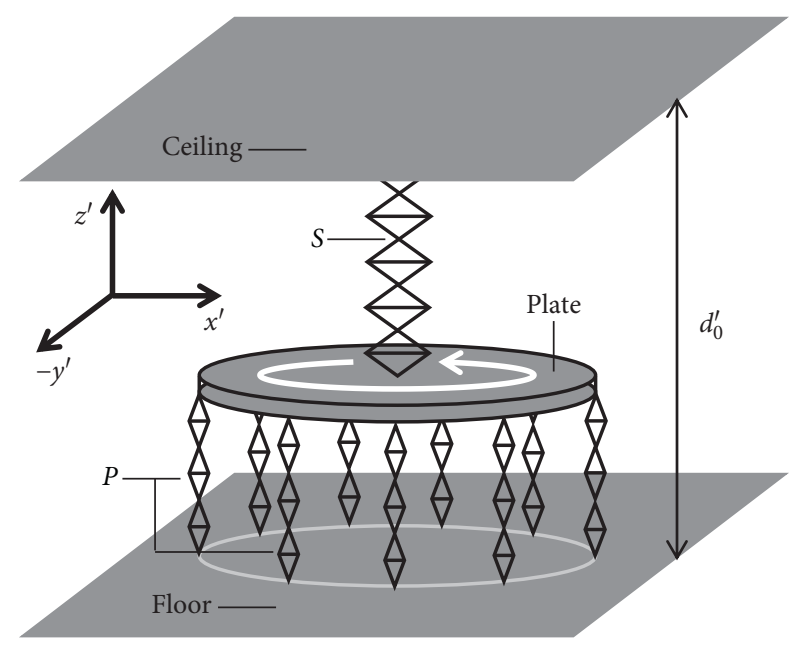

FIgURE 1: A multispring system as viewed in the lab frame of reference. The $P$ springs are set in parallel to each other and in series with $S$. The rotating plate makes the $P$ springs rotate along with it at $u^{\prime}$.

where $n^{\prime}$ is the number of thin $P$ springs. In that the thin springs are set in parallel to each other, their net constant is simply calculated to be

$$
\overline{k_{P}^{\prime \prime}}=n^{\prime} k_{P}^{\prime \prime}=n^{\prime} \frac{K_{S}^{\prime}}{n^{\prime}}=K_{S}^{\prime}
$$

In other words, the net constant of the $P$ springs is equal to that of $S$, and thus, it is anticipated that the upward forces of the $P$ springs and the downward force of $S$ are balanced, so that, from the viewpoint of the lab observer $M$, the thin plate remains motionless at a distance $d_{0}^{\prime} / 2$ from the ceiling as well as the floor level (see Figure 2(a)). Now, suppose that the plate starts to rotate about its axis of symmetry $\left(z^{\prime}\right)$ along with the thin springs $P$ fused to its perimeter. The surface of the floor is considered to be frictionless so that the other ends of the thin springs can easily slide over it, and the springs are not bent or deformed (see Figure 2(b)). If the tangential velocity $u^{\prime}$ of the cylinder's perimeter-to which the thin springs are attached-is a significant portion of light speed, the constant of each thin spring is reduced by the reciprocal of the Lorentz factor $\alpha_{u^{\prime}}[1,9]$. Therefore, we can write (see Appendix A)

$$
k_{P}^{\prime}=\alpha_{u^{\prime}} k_{P}^{\prime \prime}=\alpha_{u^{\prime}} \frac{K_{S}^{\prime}}{n^{\prime}},
$$

where $k_{P}^{\prime}$ is the reduced constant for each of the rotating thin springs measured by the lab observer $M$ and $\alpha_{u^{\prime}}=\sqrt{1-u^{\prime 2} / c^{2}}$. Moreover, it is worthwhile to mention that $k_{P}^{\prime \prime}$ is the constant for each of the $P$ springs measured either in its rest frame before the rotation, or in the frame momentarily at rest relative to the spring in the process of rotation. The net constant for the rotating springs is thus calculated as follows:

$$
\overline{k_{P}^{\prime}}=n^{\prime} k_{P}^{\prime}=n^{\prime} \alpha_{u^{\prime}} \frac{K_{S}^{\prime}}{n^{\prime}}=\alpha_{u^{\prime}} K_{S}^{\prime} .
$$

Indeed, the rotating springs are weakened due to the relativistic effects, and, as long as the springs $P$ are assembled in series with $S$, the plate finds its equilibrium state at a distance smaller than $d_{0}^{\prime} / 2$ from the floor. If we denote by $\Delta z_{S}^{\prime}$ the final displacement of $S$, the final displacement of the thin springs would then be $d_{0}^{\prime}-\Delta z_{S}^{\prime}$. When the upward force $F_{P}^{\prime}$ of the plate springs $(P)$ equals the downward force $F_{S}^{\prime}$ of $S$, the forces are in equilibrium and we have

$$
F_{S}^{\prime}=F_{P}^{\prime} \longrightarrow K_{S}^{\prime} \Delta z_{S}^{\prime}=\overline{k_{P}^{\prime}}\left(d_{0}^{\prime}-\Delta z_{S}^{\prime}\right) .
$$

Substituting Equation (4) implies

$$
\Delta z_{S}^{\prime}=\frac{\alpha_{u^{\prime}}}{1+\alpha_{u^{\prime}}} d_{0}^{\prime} .
$$

Now, we are interested in seeing if an observer $N$, who approaches the lab observer $M$ at $v$ along $x^{\prime}$, would measure $\Delta z_{S}$ the same as obtained in Equation (6) $\left(\Delta z_{S}=\Delta z_{S}^{\prime}\right)$; otherwise, relativity encounters a fatal paradox. Indeed, since the lengths in the transverse directions to the velocity $v$ are left unchanged according to the relativistic kinematics, it is expected that the measurements made by $M$ and $N$ be the same regarding the final displacement of the springs.

However, observer $N$ asserts that each thin spring travels in a trochoid curve though the resultant velocity $w$ of each $P$ spring is always perpendicular to the spring's alignment. Indeed, $N$ observes that $S$ approaches him at $v$, while each of the $P$ springs, according to their angular position in the plate, approaches or recedes from him at $w$ so that we can write

$$
w^{2}=w_{x}^{2}+w_{y}^{2}
$$

where $w_{x}$ and $w_{y}$ are the components of the resultant velocity $w$ both complying with the relativistic velocity addition formula. Therefore, if $M$ measures the velocity $u^{\prime}$ of a specific $P$ spring at an angular position of $\theta^{\prime}$ to have the components of $u_{x}^{\prime}=u^{\prime} \sin \theta^{\prime}$ and $u_{y}^{\prime}=u^{\prime} \cos \theta^{\prime} \quad$ (see Figure 3(a)), the relativistic velocity addition suggests that $N$ measures the corresponding velocities as follows [10] (see Figure 3(b)):

$$
\begin{aligned}
& w_{x}=\frac{u_{x}^{\prime}+v}{1+u_{x}^{\prime} v / c^{2}}, \\
& w_{y}=\frac{\alpha_{v} u_{y}^{\prime}}{1+u_{x}^{\prime} v / c^{2}} .
\end{aligned}
$$

Inserting Equations (8) and (9) into Equation (7), we obtain

$$
w^{2}=\frac{u^{\prime 2}+v^{2}+2 u_{x}^{\prime} v-v^{2} u_{y}^{\prime 2} / c^{2}}{\left(1+u_{x}^{\prime} v / c^{2}\right)^{2}} .
$$

Since all springs' alignments are perpendicular to the velocities of $v$ and $w$, their constants would be reduced by their corresponding reciprocal Lorentz factor. In other words, the constant of $S$ is reduced by $\alpha_{v}$ and the constants of the $P$ springs are decreased by $\alpha_{w}$ as seen by N. Now, if, for 


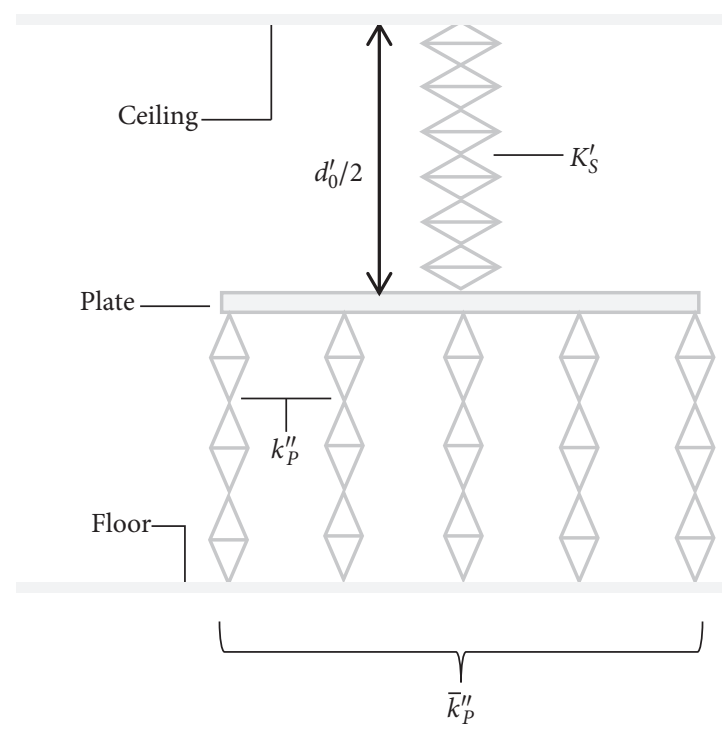

(a)

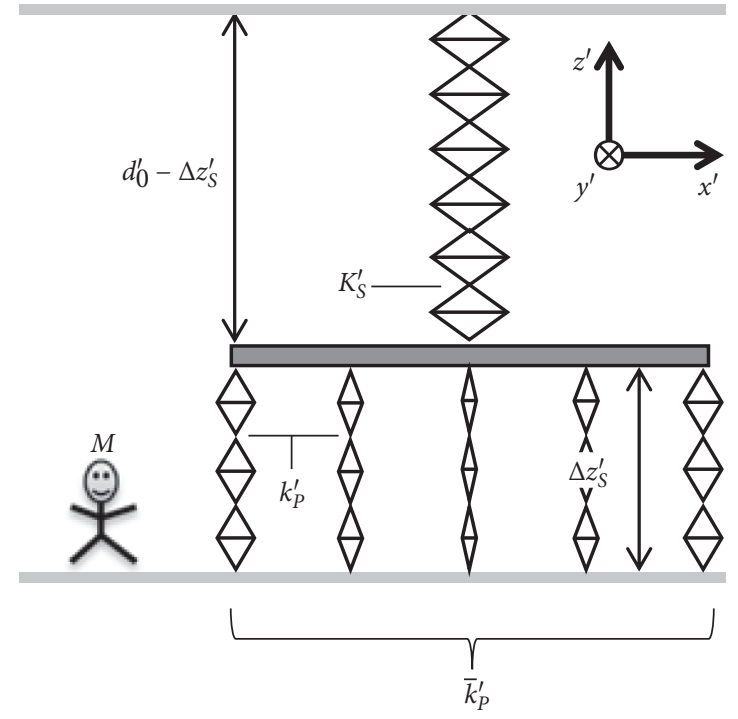

(b)

Figure 2: The multispring system as viewed in plane $x^{\prime} z^{\prime}$. (a) Before the rotation of the plate, the plate is balanced at the midpoint between the ceiling and the floor since $\overline{k_{P}^{\prime \prime}}=K_{S}^{\prime}$. (b) In the process of rotation of the plate, the $P$ springs are weakened $\left(\overline{k_{P}^{\prime}}<K_{S}^{\prime}\right)$, and thus, the plate finds its equilibrium state somewhere below the previous location. In this case, the final displacement of the $P$ springs is supposed to be $d_{0}^{\prime}-\Delta z_{S}^{\prime}$ where $\Delta z_{S}^{\prime}$ is the displacement of the spring $S$. Recall that the middle spring of the rotating plate is shown thinner in size due to maximum speed and maximum Lorentz contraction along the $x^{\prime}$-axis.

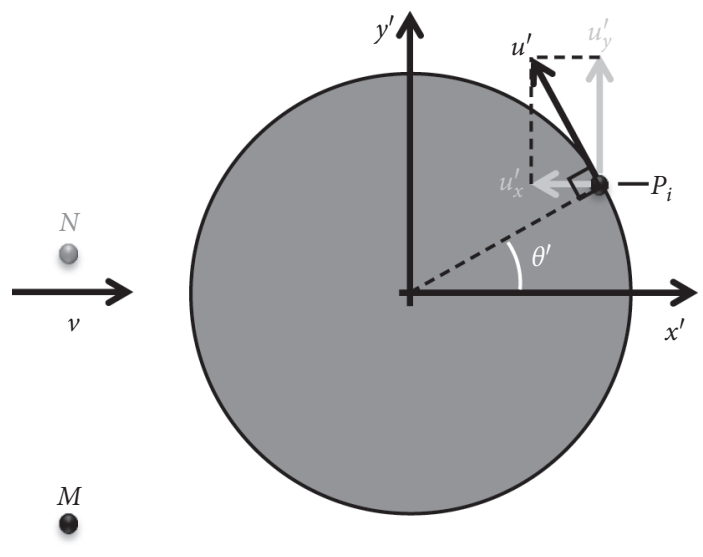

(a)

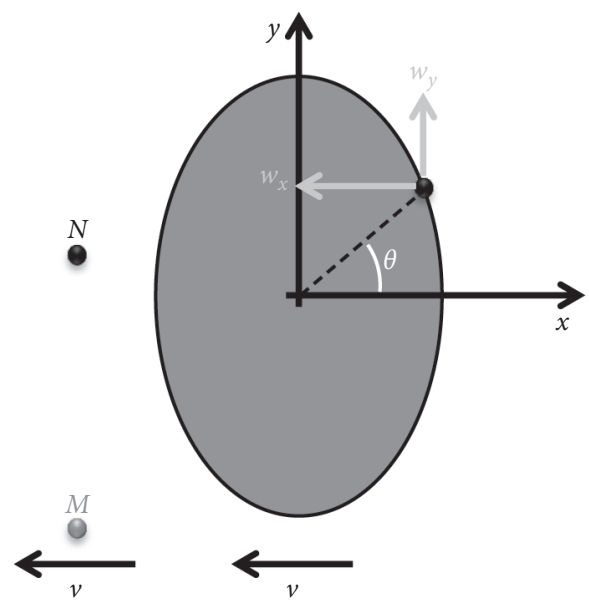

(b)

Figure 3: (a) The angular position of the $i^{\text {th }} P$ spring is shown as $P_{i}$ on the plate being observed by the lab observer $M$ in plane $x^{\prime} y^{\prime}$. Because the tangential velocity of the plate's perimeter is $u^{\prime}$, the velocity of $P_{i}$ is decomposed to $u_{x}^{\prime}$ and $u_{y}^{\prime}$. (b) The angular position of the spring as viewed by the moving observer $N$. The plate is Lorentz contracted due to its relative velocity of $v$. Indeed, $N$ asserts that the velocity $w$ of the $i^{\text {th }}$ spring has two components of $w_{x}$ and $w_{y}$ complying with the relativistic velocity addition formula.

simplicity, the number of the $P$ springs $n^{\prime}$ tends to infinity, observer $N$ can easily use integration to calculate the resultant upward force of the $P$ springs as follows:

$$
d F_{P}=d k_{P}\left(d_{0}^{\prime}-\Delta z_{S}\right)=\alpha_{w} d k_{P}^{\prime \prime}\left(d_{0}^{\prime}-\Delta z_{S}\right)
$$

where $d k_{P}^{\prime \prime}$ is the infinitesimally small constant of each of the infinite number of the $P$ springs measured either in the spring's rest frame before the rotation of the plate, or in the frame momentarily at rest with respect to the spring during the rotation. Moreover, $d k_{P}$ is the infinitesimally small constant of that specific $P$ spring as measured by $N$, and, as stated earlier, $\Delta z_{S}$ is the final displacement of $S$ measured by $N$. Remember that the displacement of the $P$ springs would then be $d_{0}^{\prime}-\Delta z_{S}$.

On the other hand, the number of the $P$ springs can be calculated by dividing the length of the plate's perimeter by the infinitesimal width of each spring: 


$$
n^{\prime}=\frac{2 \pi \alpha_{u^{\prime}} r^{\prime}}{r^{\prime} d \theta^{\prime}}=\frac{2 \pi \alpha_{u^{\prime}}}{d \theta^{\prime}}
$$

where $r^{\prime}$ is the radius of the plate measured by $M$. Recall that the plate's perimeter is Lorentz contracted by $\alpha_{u^{\prime}}$ during the rotation. Inserting Equation (12) into Equation (1), the differential form of each of the $P$ springs constant is obtained:

$$
d k_{P}^{\prime \prime}=\frac{K_{S}^{\prime}}{2 \pi \alpha_{u^{\prime}}} d \theta^{\prime}
$$

Substituting Equation (13) into Equation (11), we get

$$
d F_{P}=\left(d_{0}^{\prime}-\Delta z_{S}\right) \alpha_{w} \frac{K_{S}^{\prime}}{2 \pi \alpha_{u^{\prime}}} d \theta^{\prime},
$$

for which the integration implies

$$
F_{P}=\left(d_{0}^{\prime}-\Delta z_{S}\right) \frac{K_{S}^{\prime}}{2 \pi \alpha_{u^{\prime}}} \int_{0}^{2 \pi \alpha_{u^{\prime}}} \alpha_{w} d \theta^{\prime}
$$

Remember that it is rational to use $2 \pi \alpha_{u^{\prime}}$ instead of $2 \pi$ for the upper bound of the integrations over $\theta^{\prime}$ (see Appendix B). In that spring $S$ has a velocity $v$ from the viewpoint of $N$, its constant would reduce to $K_{S}=\alpha_{v} K_{S}^{\prime}$. The corresponding spring force would thus be

$$
F_{S}=K_{S} \Delta z_{S}=\alpha_{v} K_{S}^{\prime} \Delta z_{S}
$$

Observer $N$ claims that the upward force of $F_{P}$ should balance the downward force of $F_{S}$ in order for the plate to remain in a static situation. Using Equations (15) and (16), we have

$$
\begin{aligned}
F_{P} & =F_{S} \longrightarrow\left(d_{0}^{\prime}-\Delta z_{S}\right) \frac{K_{S}^{\prime}}{2 \pi \alpha_{u^{\prime}}} \int_{0}^{2 \pi \alpha_{u^{\prime}}} \alpha_{w} d \theta^{\prime}=\alpha_{v} K_{S}^{\prime} \Delta z_{S} \longrightarrow \\
\Delta z_{S} & =\frac{\left(1 /\left(2 \pi \alpha_{u^{\prime}}\right)\right) \int_{0}^{2 \pi \alpha_{u^{\prime}}} \alpha_{w} d \theta^{\prime}}{\alpha_{v}+\left(1 /\left(2 \pi \alpha_{u^{\prime}}\right)\right) \int_{0}^{2 \pi \alpha_{u^{\prime}}} \alpha_{w} d \theta^{\prime}} d_{0}^{\prime},
\end{aligned}
$$

where $\alpha_{w}=\sqrt{1-w^{2} / c^{2}}$. On the other hand, using Equation (10), $\alpha_{w}$ can be simplified to

$$
\alpha_{w}=\frac{\alpha_{v} \alpha_{u^{\prime}}}{1+u_{x}^{\prime} v / c^{2}}
$$

See Appendix $C$ for the proof. Substituting $u_{x}^{\prime}=u^{\prime} \sin \theta^{\prime}$ together with Equation (18) into Equation (17) yields

$$
\Delta z_{S}=\frac{(1 /(2 \pi)) \int_{0}^{2 \pi \alpha_{u^{\prime}}}\left(1+\left(u^{\prime} v \sin \theta^{\prime}\right) / c^{2}\right)^{-1} d \theta^{\prime}}{1+(1 /(2 \pi)) \int_{0}^{2 \pi \alpha_{u^{\prime}}}\left(1+\left(u^{\prime} v \sin \theta^{\prime}\right) / c^{2}\right)^{-1} d \theta^{\prime}} d_{0}^{\prime}
$$

As stated earlier, $\Delta z_{S}$ which is measured by $N$ must equal $\Delta z_{S}^{\prime}$ measured by $M$; otherwise, relativity results in a paradox. Comparing Equation (19) with Equation (6), if $\Delta z_{S}=\Delta z_{S}^{\prime}$, we indeed get

$$
\frac{1}{2 \pi} \int_{0}^{2 \pi \alpha_{u^{\prime}}} \frac{d \theta^{\prime}}{1+\left(u^{\prime} v \sin \theta^{\prime}\right) / c^{2}}=\alpha_{u^{\prime}} .
$$

Unfortunately, the above formula is not always valid for all arbitrary values of $u^{\prime}$ and $v$, and thus, it seems that relativity includes a null result, at least, in this example. To prove, it suffices to substitute $v=0.6 c$ and $u^{\prime}=0.8 c$ and do the calculations numerically. In this case, the left-hand side of Equation (20) equals 0.506, whereas the right-hand side equals 0.600 , which are not equal to each other. This counterexample shows a deficit in special relativity. However, one also can take the integral analytically to show that Equation (20) is not valid for all arbitrary values of $u^{\prime}$ and $v$.

An important point with this problem is that if the forces are transmitted via some sort of signaling from the $P$ springs towards the center of rotation of the plate to which one end of $S$ is attached, the arrival of the signals to the center is simultaneous from the viewpoint of both $M$ and $N$. This simultaneity makes the spring $S$ react to all of the signals sent by the $P$ springs instantly as viewed by both of the observers; otherwise, it is expected that the plate is deformed in shape due to the signal delays.

\section{Important Notes regarding This Paradox}

To reduce the reader's confusion, we gather some important remarks concerning this problem and the possible resolutions:

(1) Remember that this problem is not connected to some aspects of the Ehrenfest paradox [11] according to which a fast-rotating disc cannot approach the light speed since the centrifugal pressure exceeds the shear modulus of the material of which the plate is made. In our problem, indeed, it is not necessary for the tangential velocity $u^{\prime}$ of the plate to have a value close to $c$ in order to encounter a paradox. That is, if $u^{\prime}$ is much smaller than the speed of light, the paradox is still valid though the difference in $\Delta z$ and $\Delta z^{\prime}$ is very small.

(2) The centrifugal force exerted on the $P$ springs due to the rotation of the plate may bend the springs slightly outward the center of rotation; however, this phenomenon can be neglected by assuming that the said force is not great, or the springs' stiffness is great so that they are not easily bent out of shape.

(3) The uniform distribution of the $P$ springs as seen by $M$ is no longer uniform from the viewpoint of $N$ (see Figure 4). Remember that this phenomenon has already been discussed in the literature [12]; however, it is unlikely that this nonuniformity can cause the plate's normal to incline relative to the $z$ direction as viewed by $N$. In fact, one can claim that the resultant force of the upper springs would certainly balance that of the lower ones measured by $M$ (see Figure 4(a)) such that the plate remains parallel to the floor level, whereas $N$ may claim that, due to the nonuniform distribution of the $P$ springs, the mentioned forces are not balanced, which makes the plate become oblique (see Figure 4(b)). Recall that if the plate is inclined in the rest frame of $N$, in that it is not measured inclined in that of $M$, this can bring 


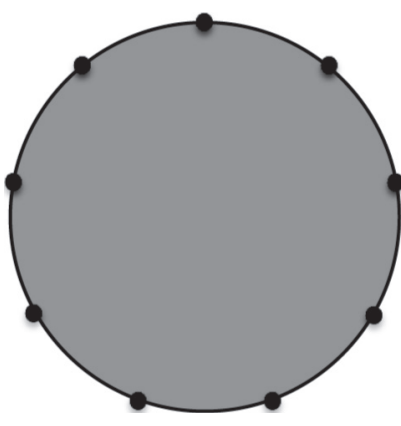

(a)

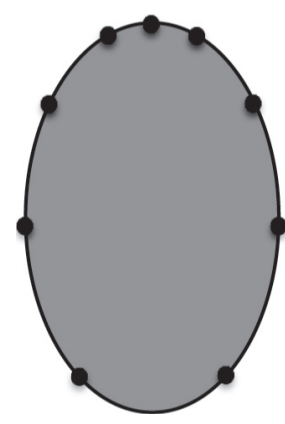

(b)

Figure 4: (a) The distribution of the $P$ springs looks uniform from the viewpoint of the lab observer $M$. (b) The distribution is no longer uniform as viewed by the moving observer $N$. The density of the $P$ springs' distribution, as well as the mass density of the plate, is higher where the tangential velocity of the plate $(w)$ is greater, and vice versa.

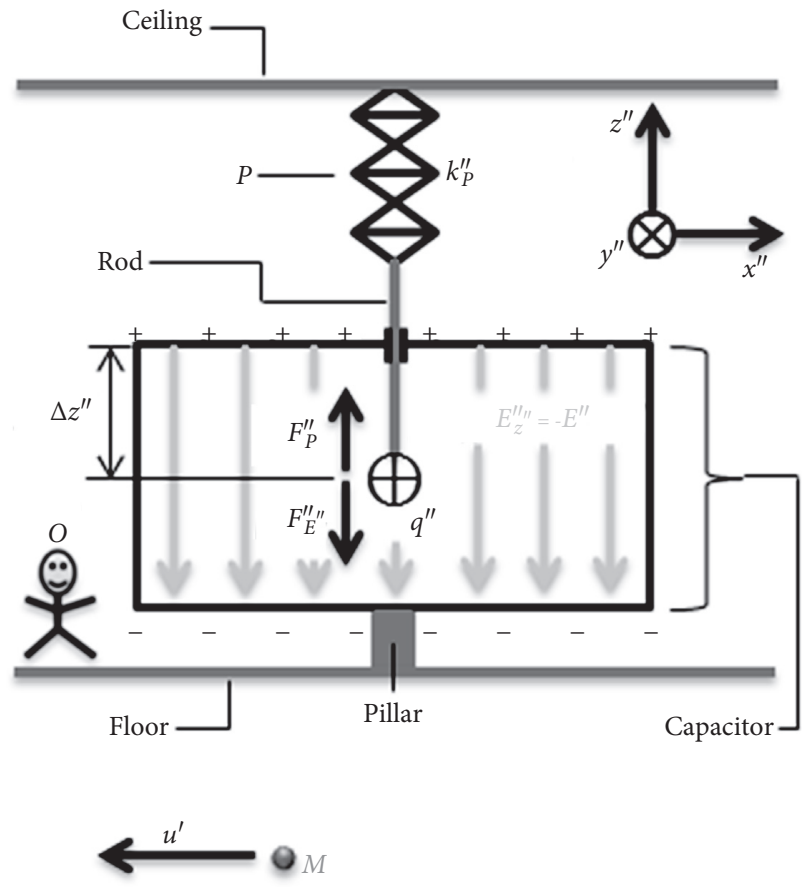

Figure 5: The spring $P$ is attached to a thin, frictionless rod, which has pierced the upper plate of a parallel-plate capacitor. Since the spring force of $F_{P}^{\prime \prime}$ cancels out the field force of $F_{E^{\prime \prime}}^{\prime \prime}$, the tiny charge $q^{\prime \prime}$, which is attached to the lower end of the rod, is suspended statically from the standpoint of the lab observer $O$. Both the rod and the pillar are made of nonconductive materials. On the other hand, because the spring is supposed to be located far outside the capacitor, no EM field affects it. Observer $M$ moves at $u^{\prime}$ along $-x^{\prime \prime}$ as viewed by $O$.

about another paradox besides the main paradox discussed earlier. However, the author guesses that the increase in the density of the springs takes place for those having greater tangential speeds from N's point of view, and thus, the spring constants would have smaller values. On the other hand, the lesser the springs' density, the slower they move and the greater their constants. Therefore, it is possible that the increase in springs' density compensates for the decrease in their constants, and vice versa, so that the upper and lower resultant forces would finally balance each other, which prohibits the plate from additional rotation. It is also possible that this inclination is somehow related to the disputatious arguments about Mansuripur's article where a similar nonuniformity in the distribution of some point-like electrical charges causes the moving observer to detect a possible torque on a current-carrying loop of wire, whereas the lab observer does not, due to the uniform distribution of the charges [13-16]. A comprehensive discussion is beyond the scope of this article.

(4) It is not mandatory to consider an infinite number of the $P$ springs. One can repeat the calculations using any finite number of springs.

(5) Instead of involving the viewpoint of the observer in the rest frame of the $P$ springs, one can directly apply the Lorentz transformation for force between $M$ and $N$ to finally reach Equation (20) (see Appendix D).

(6) Remember that this article does not question the relativistic version of Hook's law, but rather the relativistic transformation for force in its general form. Hence, one can replace the springs with electromagnetic fields and electrical charges in a way similar to [2] in order to rewrite the paradox (see Appendix D).

\section{Conclusion}

As a complementary to the author's previous works regarding the relativistic force transformation, this article shows, too, an inconsistency between the kinematics and dynamics in relativity.

\section{Appendix}

\section{A. Derivation of Equation (3)}

We give here an explicit derivation of Equation (3). Assume that a thin nonconductive rod has pierced the positively charged plate of a parallel-plate capacitor inside which there 
is a uniform electric field of $E_{z^{\prime \prime}}^{\prime \prime}=-E^{\prime \prime}$. The rod is attached to a spring $(P)$ with a constant of $k_{P}^{\prime \prime}$ in one end, and in the other end, it is attached to a point-like, positively charged object $\left(q^{\prime \prime}\right)$. It is assumed that the rod is frictionless and can thus easily move up and down along $z^{\prime \prime}$. Both the spring and the charged object are considered massless, and the experiment is carried out away from any gravitational field. The spring in turn is attached to the ceiling of the lab in the upper end (see Figure 5).

If the spring is in its free length position $\left(E_{z^{\prime \prime}}^{\prime \prime}=0\right)$, it is supposed that the tiny charge $q^{\prime \prime}$ is located very close to the positively charged plate of the capacitor. Therefore, when the capacitor is charged, the spring is stretched with a displacement of $\Delta z^{\prime \prime}$ until the electrical force of the field $\left(F_{E^{\prime \prime}}^{\prime \prime}\right)$ cancels out the spring force of $F_{P}^{\prime \prime}$. In fact, when the oscillations damp out, $q^{\prime \prime}$ finds its equilibrium state at $\Delta z^{\prime \prime}$ from the upper plate as seen by the lab observer, which, this time, we denote by $O$. We thus can write the following:

$$
\left|F_{E^{\prime \prime}}^{\prime \prime}\right|=\left|F_{P}^{\prime \prime}\right| \longrightarrow E^{\prime \prime} q^{\prime \prime}=k_{P}^{\prime \prime} \Delta z^{\prime \prime} .
$$

We are now interested to find the spring constant of $k_{P}^{\prime}$ from the standpoint of the moving observer $M$ relative to which observer $O$, as well as the system of spring-capacitor, indeed, moves along $+x^{\prime}$ at $u^{\prime}$. Using the Lorentz transformation for EM fields, observer $M$, however, detects a magnetic field of $B_{y^{\prime}}^{\prime}=\gamma_{u^{\prime}}\left(B_{y^{\prime \prime}}^{\prime \prime}-u^{\prime} E_{z^{\prime \prime}}^{\prime \prime} / c^{2}\right)$ besides an electric field of $E_{z^{\prime}}^{\prime}=\gamma_{u^{\prime}}\left(E_{z^{\prime \prime}}^{\prime \prime}-u^{\prime} B_{y^{\prime \prime}}^{\prime \prime}\right)([10]$ p. 166). Inasmuch as $B_{y^{\prime \prime}}^{\prime \prime}=0$ and $E_{z^{\prime \prime}}^{\prime \prime}=-E^{\prime \prime}$, we have

$$
\begin{aligned}
B_{y^{\prime}}^{\prime} & =u^{\prime} \gamma_{u^{\prime}} E^{\prime \prime} / c^{2}, \\
E_{z^{\prime}}^{\prime} & =-\gamma_{u^{\prime}} E^{\prime \prime} .
\end{aligned}
$$

Equation (A.2) infers the corresponding Lorentz force of $F_{B^{\prime}}^{\prime}=q^{\prime \prime} u^{\prime} B_{y^{\prime}}^{\prime}=\gamma_{u^{\prime}} E^{\prime \prime} q^{\prime \prime} u^{\prime 2} / c^{2}$, which is exerted on $q^{\prime \prime}$ along $+z^{\prime}$, and Equation (A.3) implies an electric force of $F_{E^{\prime}}^{\prime}=q^{\prime \prime} E_{z^{\prime}}^{\prime}=-\gamma_{u^{\prime}} E^{\prime \prime} q^{\prime \prime}$, which is exerted on $q^{\prime \prime}$ along $-z^{\prime}$. The resultant force of $F_{E^{\prime}-B^{\prime}}^{\prime}$ due to the EM fields is calculated as follows:

$$
\begin{aligned}
& F_{B^{\prime}-E^{\prime}}^{\prime}=F_{B^{\prime}}^{\prime}+F_{E^{\prime}}^{\prime}=\left(\gamma_{u^{\prime}} E^{\prime \prime} q^{\prime \prime} u^{\prime 2} / c^{2}\right)+\left(-\gamma_{u^{\prime}} E^{\prime \prime} q^{\prime \prime}\right) \longrightarrow \\
& F_{B^{\prime}-E^{\prime}}^{\prime \prime}=-\alpha_{u^{\prime}} E^{\prime \prime} q^{\prime \prime} .
\end{aligned}
$$

On the other hand, observer $M$ calculates the spring force to be

$$
F_{P}^{\prime}=k_{P}^{\prime} \Delta z^{\prime} .
$$

Because $M$, as well as $O$, admits the static situation of $q^{\prime \prime}$, the above forces shall cancel out each other, and hence, we have

$$
\left|F_{B^{\prime}-E^{\prime}}^{\prime}\right|=\left|F_{P}^{\prime}\right| \longrightarrow \alpha_{u^{\prime}} E^{\prime \prime} q^{\prime \prime}=k_{P}^{\prime} \Delta z^{\prime} \longrightarrow k_{P}^{\prime}=\frac{\alpha_{u^{\prime}} E^{\prime \prime} q^{\prime \prime}}{\Delta z^{\prime}} .
$$

Moreover, the traditional Lorentz transformation asserts that the lengths perpendicular to the motion direction are left unchanged, otherwise paradoxes arise. Therefore, we have

$$
\Delta z^{\prime}=\Delta z^{\prime \prime} .
$$

Substituting Equation (A.1) together with Equation (A.7) into Equation (A.6) yields

$$
k_{P}^{\prime}=\alpha_{u^{\prime}} k_{P}^{\prime \prime}
$$

Equation (3) is thus proved explicitly.

\section{B. Regarding the Upper Bound of the Integrations}

It is evident that the number of springs must remain the same before and during the rotation $\left(n^{\prime \prime}=n^{\prime}\right)$. That is to say, the number of springs is independent of whether or not the Lorentz contraction occurs. Before the rotation, observer $M$ calculates this number to be

$$
n^{\prime \prime}=\frac{2 \pi r^{\prime}}{r^{\prime} d \theta^{\prime \prime}}=\frac{2 \pi}{d \theta^{\prime \prime}}
$$

Now, if we equate Equation (B.1) with Equation (12), we get

$$
n^{\prime \prime}=n^{\prime} \longrightarrow d \theta^{\prime}=\alpha_{u^{\prime}} d \theta^{\prime \prime} .
$$

The integration implies

$$
\theta^{\prime}=\alpha_{u^{\prime}} \theta^{\prime \prime}+C,
$$

where $C$ is the integration constant. This constant can be chosen to be zero inasmuch as for $\theta^{\prime}=0$, we set $\theta^{\prime \prime}=0$. Therefore, we have

$$
\theta^{\prime}=\alpha_{u^{\prime}} \theta^{\prime \prime}
$$

It is evident that a complete period occurs on the interval $(0,2 \pi)$ for $\theta^{\prime \prime}$ as measured by $M$ before the rotation. To find the upper bound of all integrations over $\theta^{\prime}$, it suffices to insert $\theta^{\prime \prime}=2 \pi$ into Equation (B.4):

$$
\theta^{\prime}=\alpha_{u^{\prime}} 2 \pi .
$$

On the other hand, in the last appendix, we have introduced an alternative approach to this paradox, which does not involve using spring constants or the Lorentz contraction directly. There, another method is demonstrated for proving the use of $2 \pi \alpha_{u^{\prime}}$ instead of $2 \pi$ as the upper bound of the said integrations (see Appendix D).

\section{Derivation of Equation (18)}

Equation (18) is proved in this appendix. Using Equation (10), we can write 


$$
\begin{aligned}
\alpha_{w} & =\sqrt{1-w^{2} / c^{2}}=\sqrt{1-\frac{u^{\prime 2}+v^{2}+2 u_{x}^{\prime} v-v^{2} u_{y}^{\prime 2} / c^{2}}{\left(1+u_{x}^{\prime} v / c^{2}\right)^{2} c^{2}}} \\
& =\sqrt{\frac{\left(1+u_{x}^{\prime} v / c^{2}\right)^{2} c^{2}}{\left(1+u_{x}^{\prime} v / c^{2}\right)^{2} c^{2}}-\frac{u^{\prime 2}+v^{2}+2 u_{x}^{\prime} v-v^{2} u_{y}^{\prime 2} / c^{2}}{\left(1+u_{x}^{\prime} v / c^{2}\right)^{2} c^{2}}} \\
& =\frac{1}{\left(1+u_{x}^{\prime} v / c^{2}\right) c} \times \sqrt{\left(1+u_{x}^{\prime} v / c^{2}\right)^{2} c^{2}-\left(u^{\prime 2}+v^{2}+2 u_{x}^{\prime} v-v^{2} u_{y}^{\prime 2} / c^{2}\right)} \\
& =\frac{1}{\left(1+u_{x}^{\prime} v / c^{2}\right) c} \times \sqrt{c^{2}+\frac{u_{x}^{\prime 2} v^{2}}{c^{2}}+2 u_{x}^{\prime} v-u^{\prime 2}-v^{2}-2 u_{x}^{\prime} v+\frac{u_{y}^{\prime 2} v^{2}}{c^{2}}} \\
& =\frac{1}{\left(1+u_{x}^{\prime} v / c^{2}\right) c} \sqrt{c^{2}-u^{\prime 2}+\frac{\left(u_{x}^{\prime 2}+u_{y}^{\prime 2}\right) v^{2}}{c^{2}}-v^{2} .}
\end{aligned}
$$

Recall that the cross sign " $x$ " indicates the usual multiplication rather than the vector product. Considering the fact that $u^{\prime 2}=u_{x}^{\prime 2}+u_{y}^{\prime 2}$, we continue

$$
\begin{aligned}
\alpha_{w} & =\frac{1}{\left(1+u_{x}^{\prime} v / c^{2}\right) c} \sqrt{c^{2}-u^{\prime 2}+\frac{u^{\prime 2} v^{2}}{c^{2}}-v^{2}} \\
& =\frac{1}{1+u_{x}^{\prime} v / c^{2}} \sqrt{1-\frac{u^{\prime 2}}{c^{2}}+\frac{u^{\prime 2} v^{2}}{c^{4}}-\frac{v^{2}}{c^{2}}} \\
& =\frac{1}{1+u_{x}^{\prime} v / c^{2}} \sqrt{\left(1-\frac{u^{\prime 2}}{c^{2}}\right)-\frac{v^{2}}{c^{2}}\left(1-\frac{u^{\prime 2}}{c^{2}}\right)} \\
& =\frac{1}{1+u_{x}^{\prime} v / c^{2}} \sqrt{\left(1-\frac{u^{\prime 2}}{c^{2}}\right)\left(1-\frac{v^{2}}{c^{2}}\right)} \\
& =\frac{1}{1+u_{x}^{\prime} v / c^{2}} \sqrt{1-\frac{u^{\prime 2}}{c^{2}} \sqrt{1-\frac{v^{2}}{c^{2}}}}
\end{aligned}
$$

which finally yields

$$
\alpha_{w}=\frac{\alpha_{v} \alpha_{u^{\prime}}}{1+u_{x}^{\prime} v / c^{2}}
$$

Therefore, Equation (18) is proved.

\section{Eliminating the Use of Spring Constants}

Here, not only we directly use the Lorentz transformation for force to relate the viewpoints of $M$ and $N$, but we eliminate the use of spring constants. Assume that we replace the spring $S$ shown in Figure 1 with a cylinder inside which there is a uniform electric field. It is supposed that an electrically charged object acts as a piston inside this cylinder. (In Figure 5, if we eliminate the spring $P$, the remaining capacitor is similar to a cylinder inside which the charged object $\left(q^{\prime \prime}\right)$ and the rod behave as a piston, which very well depicts our purpose.)

Indeed, we have produced some sort of spring, which can exert a constant force regardless of the displacement of the charged piston. If the $P$ springs, in the article's main problem (see Figure 1), are also replaced by some similar cylinders, though each being very thinner in size and having an infinitesimally small charged piston, the spring $S$ and each of the $P$ springs, respectively, exert the forces of $F_{S}^{\prime}$ and $d F_{P}^{\prime}$ from the standpoint of observer $M$. Now, if $M$ claims that the system is balanced and thus the forces cancel out each other, we can write

$$
F_{S}^{\prime}=\int d F_{P}^{\prime}
$$

The Lorentz transformation for force asserts that $N$ calculates the corresponding forces as follows ([10] p. 147):

$$
F_{S}=\alpha_{v} F_{S}^{\prime}
$$

$$
d F_{P}=\frac{\alpha_{v} d F_{P}^{\prime}}{1+u_{x}^{\prime} v / c^{2}} .
$$

If relativity excludes any null result, $N$ would also claim that the forces would balance each other; otherwise, the plate would accelerate upward or downward along $z$. Therefore, the static situation implies

$$
F_{S}=\int d F_{P}
$$

Substituting Equations (D.2) and (D.3) into Equation (D.4), we obtain 


$$
\alpha_{v} F_{S}^{\prime}=\int \frac{\alpha_{v} d F_{P}^{\prime}}{1+u_{x}^{\prime} v / c^{2}} .
$$

Inasmuch as $d F_{P}^{\prime}$ is independent of $v$ and $u_{x}^{\prime}$, we can write

$$
\alpha_{v} F_{S}^{\prime}=\frac{\alpha_{v}}{1+u_{x}^{\prime} v / c^{2}} \int d F_{P}^{\prime}
$$
yields

Finally, inserting Equation (D.1) into Equation (D.6)

$$
\alpha_{v} F_{S}^{\prime}=\frac{\alpha_{v}}{1+u_{x}^{\prime} v / c^{2}} F_{S}^{\prime} \longrightarrow 1=\frac{1}{1+u_{x}^{\prime} v / c^{2}} .
$$

Since Equation (D.7), similar to Equation (20), has two unacceptable solutions of $v=0$ or $u_{x}^{\prime}=0 \longrightarrow u^{\prime}=0$, it shows that observer $N$, contrary to $M$, believes that the plate would accelerate along $z$. Remember that if we first insert $u_{x}^{\prime}=u^{\prime} \sin \theta^{\prime}$ into Equation (D.7) and then integrate both sides of Equation (D.7) with respect to $\theta^{\prime}$ from 0 to $2 \pi \alpha_{u^{\prime}}$, we reach exactly Equation (20).

Remember that these calculations are also applicable to the original problem including springs provided the difference of the final displacements of the springs measured by $M$ and $N$ are small so that the related forces remain nearly unchanged. On the other hand, we are not worried about how the electromagnetic fields change as viewed by $N$ in this later example because whatsoever they are, they must produce the resultant forces complying with the Lorentz transformation for force.

Therefore, it is also possible to use, instead of springs, a cylinder filled with an ideal gas along with a moveable piston regardless of the type of the thermodynamic process according to which the piston compresses/decompresses the gas contained within the cylinder and regardless of how the thermodynamic parameters such as temperature and pressure are defined relativistically. It is because the calculations done in this appendix are general for all forces, which can be applied to any problem regardless of the agent(s) of the involved force(s).

\section{Data Availability}

Data sharing is not applicable to this article as no new data were created or analyzed in this study.

\section{Conflicts of Interest}

The author declares no conflicts of interest.

\section{Acknowledgments}

The author would like to express gratitude to Mr. Amir Assarzadeh for reviewing the calculations and reminding him of the fact that the nonuniform distribution of the springs may compensate for the changes in the spring constants so that the plate may not represent additional rotation from the viewpoint of the observers.

\section{References}

[1] M. Javanshiry, "Is there any rational transformation for the relativistic force?" Annals of the "Dunarea de Jos" University of Galati. Fascicle II, Mathematics, Physics, Theoretical Mechanics, vol. 43, no. 2, pp. 170-174, 2020.

[2] M. Javanshiry, "An ambiguity in the relativistic dynamics and electromagnetism regarding force transformation," Bulletin of Pure \& Applied Sciences-Physics, vol. 39D, no. 2, pp. 140-144, 2020.

[3] W. Nawrot, "The Hafele-Keating paradox-Serious problems of the special theory of relativity?" Physics Essays, vol. 27, no. 4, pp. 598-600, 2014.

[4] R. Szostek, "Derivation method of numerous dynamics in the special theory of relativity," Open Physics, vol. 17, no. 1, pp. 153-166, 2019.

[5] G. M. Koczan, "New definitions of 3D acceleration and inertial mass not violating $\mathrm{F}=\mathrm{MA}$ in the special relativity," Results in Physics, vol. 24, Article ID 104121, 2021.

[6] G. M. Koczan, "Relativistic relative velocities and relativistic acceleration," Acta Physica Polonica A, vol. 139, no. 4, pp. 401-406, 2021.

[7] K. Szostek and R. Szostek, "Kinematics in the special theory of ether," Moscow University Physics Bulletin, vol. 73, no. 4, pp. 413-421, 2018.

[8] R. Szostek, "Derivation of all linear transformations that meet the results of Michelson-Morley experiment and discussion of the relativity basics," Moscow University Physics Bulletin, vol. 75, no. 6, pp. 684-704, 2020.

[9] Ø. Grøn, "Covariant formulation of Hooke's law," American Journal of Physics, vol. 49, pp. 28-30, 1981.

[10] R. Resnick, Introduction to Special Relativity, p. 83, Wiley, New York, NY, USA, 1968.

[11] P. Ehrenfest, "Gleichförmige rotation starrer körper und relativitätstheorie," Physikalische Zeitschrift, vol. 10, p. 918, 1909.

[12] V. N. Matvejev, O. V. Matvejev, and Ø. Grøn, "A relativistic trolley paradox," American Journal of Physics, vol. 84, no. 6, pp. 419-425, 2016.

[13] M. Mansuripur, "Trouble with the Lorentz law of force: incompatibility with special relativity and momentum conservation," Physical Review Letters, vol. 108, no. 19, Article ID 193901, 2012.

[14] D. J. Griffiths and V. Hnizdo, "Mansuripur's paradox," American Journal of Physics, vol. 81, no. 8, pp. 570-574, 2013.

[15] M. Mansuripur, "Trouble with the Lorentz law of force: response to critics," Proceedings of SPIE, vol. 8455, Article ID 845512, 2012.

[16] F. Redfern, "An alternative resolution to the Mansuripur paradox," Physica Scripta, vol. 91, no. 4, Article ID 045501, 2016. 